\title{
A Siphon-based Deadlock Prevention Policy for a Class of Petri Nets - $S^{3}$ PMR
}

\author{
Mingming Yan, Rongming Zhu, Zhiwu Li and Mengchu Zhou
School of Electro-Mechanical Engineering, Xidian University, Xi'an 710071 P. R. China (Tel: +86-29-88-20-24-60;
e-mail:yanmingstar@gmail.com,email:zhu.rm@163.com,e-mail: systemscontrol@gmail.com,e-mail:arwang@mail.xidian.edu.cn) Department of Electrical and Computer Engineering, New Jersey Institute of Technology Newark, NJ 07102, USA (zhou@njit.edu)

\begin{abstract}
This paper focuses on the problem of deadlocks in automated flexible manufacturing systems (FMS) where deadlocks are caused by unmarked siphons in their Petri net models. A deadlock prevention policy is proposed for a subclass of Petri nets, $\mathrm{S}^{3} \mathrm{PMR}$ that can well model a large class of FMS. We distinguish siphons in such a net model by elementary and dependent ones. For each elementary siphon, a monitor is added to the plant model such that it is invariantcontrolled. The monitor addition way guarantees that no emptiable control-induced siphon in the resultant net is generated due to the addition of monitors. This novel deadlock prevention policy can usually lead to a more permissive supervisor by adding a relatively much smaller number of monitors and arcs than the existing methods for the design of liveness-enforcing Petri net supervisors. Experimental study validates the result.
\end{abstract}

\section{INTRODUCTION}

A deadlock occurs in a flexible manufacturing system (FMS) when parts are blocked and waiting for resources held by others that will never be granted. One way of dealing with deadlock problems is, first, to model an FMS with Petri nets Murata [1989], Zhou and Kurapati [1999], and Hruz and Zhou [2007]. Three basic approaches are used to resolve deadlock problems Zhou and Fanti [2005]. The first is called deadlock detection and recovery Wysk et al. [1991]. A deadlock detection approach permits deadlock to occur and does not solve it. Once a deadlock state has been detected, deadlocks are recovered by pre-emptying some of the resources involved in them. The second strategy, namely deadlock avoidance, projects deadlock detection into the future in order to keep the system from committing itself to an allocation that may eventually lead to a deadlock Banaszak and Krogh [1990], Hsien and Chang [1994], Xing et al. [1996], Park and Reveliotis [2001], and Abdallah and ElMaraghy [1998]. Such a strategy, unfortunately, may not eliminate deadlocks completely. Finally, the third approach called deadlock prevention is either to design a system such that deadlocks will never occur or to add a control mechanism on resource requests which prevents deadlocks from occurring.

In FMS context, deadlock prevention is usually achieved either by effective system design or by using an off-line mechanism to control the requests for resources to ensure that deadlocks never occur Xie and Peng [2002] and Zhou and Dicesare [1993]. Monitors or control places and related arcs are often used to achieve such purposes Ezpeleta et al. [1995], Abdallah and ElMaraghy [1998], Li and Zhou [2004], Huang et al. [2001], Chao [2006], Uzam [2002], and Uzam [2004]. The work of Ezpeleta et al. [1995] is usually considered to be the first using structure theory of Petri nets to design monitor-based liveness-enforcing Petri net supervisors for FMS. They defined a subclass of ordinary and conservative Petri nets called System of Simple Sequential Processes with Resources $\left(S^{3} \mathrm{PR}\right)$ and required the target Petri net to be in that subclass. A monitor is added to every strict minimal siphon such that liveness can be enforced. However, too many monitors and arcs have to be added, leading to a much more complex Petri net supervisor than the originally built Petri net model. Furthermore, the behavior of the system can be rather restrictive.

A deadlock prevention policy is proposed in this paper based on the structure analysis of Petri nets for a class of petri nets that is broader than $\mathrm{S}^{3} \mathrm{PR}$, called $\mathrm{S}^{3} \mathrm{PMR}$, where deadlocks are related to the unmarked siphons. We first distinguish siphons in such a plant Petri net model by elementary and dependent ones. Then, for each elementary siphon, a monitor is added to the plant model such that the siphon is invariant-controlled. The way to add monitors does not lead to unmarked control-induced siphons in the resultant net. By designing the control depth variables for elementary siphons, dependent siphons can be fully controlled, which leads to a liveness-enforcing Petri net supervisor. It is emphasized that the original net is ordinary and allows more than one different shared resource at each operation stage. Barkaoui and PradatPeyre [1996] proposed the concepts of max cs-property and min cs-property. Because the original nets are all ordinary in this paper, we have $\max _{p} \bullet=1$. It is shown that if a marked net is invariant-controlled by adding monitor $V_{S}$, then it satisfies the max-controlled siphon property (max cs-property). 
The rest of this paper is organized as follows. Section 2 reviews preliminaries of Petri nets that are used throughout the paper. The deadlock control policy is proposed in Section 3. Section 4 introduces an FMS example to illustrate the applications of the proposed policy. Finally, Section 5 concludes this paper.

\section{PRELIMINARIES}

As for the standard definitions of deadlock-freeness, liveness, reversibility, and boundedness, the reader is referred to Murata [1989]. Elementary siphons of Petri nets are proposed in Li and Zhou [2004].

Our deadlock prevention policy targets the system modeled by a class of ordinary Petri nets called $\mathrm{S}^{3} \mathrm{PMR}$. This section introduces $\mathrm{S}^{3} \mathrm{PMR}$ and RCN-merged net models. An $\mathrm{S}^{3} \mathrm{PMR}$ is defined as follows Huang et al. [2006].

Definition 1. A process net is a strongly connected state machine $(P, T, F, W)$ with exactly one initially marked place $p^{0}$ (idle place) such that each circuit of the net contains $p^{0}$. The other places are called operation places.

Definition 2. An $\mathrm{S}^{3} \mathrm{PMR}$ net $N$ is a net that results from adding a set $R$ of initially marked places (resource places) to a set of independent process nets.

1) Each resource place $r$ is associated with a set of operation places, $O P(r)$. This implies that these operation places require $r$.

2) For each transition $t$, which satisfies $t \in{ }^{\bullet} p$ of some $p \in O P(r)$, there exists an arc from $r$ to $t$ if $\bullet \cap O P(r)=\emptyset$.

3) For each transition $t$, which satisfies $t \in p^{\bullet}$ of some $p \in O P(r)$, there exists an arc from $t$ to $r$ if $t^{\bullet} \cap O P(r)=\emptyset$.

An $\mathrm{S}^{3} \mathrm{PR}$ is an $\mathrm{S}^{3} \mathrm{PMR}$, in which each operation place is associated with an unique resource place, and two consecutive operation places are associated with different resource places.

Let $x$ and $y$ be two nodes of an $S^{3} P M R$ net $N$. We will say that $x$ is previous to $y$ in $N$ if and only if there exists an elementary path in a circuit $C$ in $N$ such that its length, i.e., the number of nodes, of which is greater than 1 and it does not contain $p_{i}^{0}$. This fact is denoted by $x<_{N} y$, and the simple path from $x$ to $y$, denoted by $S P(x, y)$.

Definition 3. Let $N=\bigcirc_{i=1}^{k} N_{i}=\left(P \cup P^{0} \cup P_{R}, T, F\right)$ be an $\mathrm{S}^{3} \mathrm{PMR}$ and $S$ be a strict minimal siphon in $N$, where $S=S_{P} \cup S_{R}, S_{R}=S \cap P_{R}$, and $S_{P}=S \backslash S_{R}$. Let $[S]=\left(\cup_{r \in S_{R}} O P(r)\right) \backslash S$. [S] is called the complementary set of siphon $S$, and $W_{p}=\max [S]\left(p^{\prime}\right)\left\{p^{\prime} \mid p^{\prime} \in S P\left(p, p_{i}^{0}\right) \cap\right.$ $\left.P_{i}\right\}$. If $q \in S P\left(p, p_{i}^{0}\right) \cap P_{i}$, then $W_{q} \leq W_{p}$.

From the above definition, it is easy to see that $W_{p}$ means the maximal number of tokens derived from siphon $S$ to complete the process $i$ when a token arrives at place $p$ $\left(p \in P_{i}\right)$.

In addition, an $\mathrm{S}^{3} \mathrm{PMR}$ has the following properties. (1) $\forall i \in\{1,2, \ldots, k\}, P_{i} \cup\left\{p_{i}^{0}\right\}$ is the support of a P-semiflow; and $\forall r \in P_{R}, O P(r) \cup\{r\}$ is the support of a P-semiflow. (2) Given a strict minimal siphon $S$ in $N,[S] \cup S$ is the support of a $P$-semiflow.

Definition 4. A Resource Control Net (RCN) is a strongly connected state machine $\left(P, T, F, M_{0}\right)$ in which there exists one and only one place $p_{r} \in P$, called a resource place, such that $M_{0}\left(p_{r}\right) \neq 0$. The remaining places are called operation places.

By construction, an RCN-merged net is State Machine Decomposable. Each RCN is a state machine component. The following restrictions concerning the merge of RCN's are required.

Restriction 1: At each common transition, there exists at most one input place that is an operation place.

Restriction 2: Common transition subnet should not include resource places.

Restriction 3: The Petri net $N^{*}$ derived from the integrated model $N$ by removing the resource places is an acyclic graph.

Restriction 4: At any common transition, there is at most one output place that is an operation place.

Theorem 1. Suppose that Restrictions 1-4 hold. Then an RCN-merged net is live and reversible if and only if no siphon can become empty Jeng and Xie [2004].

Theorem 2. Each $\mathrm{S}^{3} \mathrm{PMR}$ is an RCN-merged net Huang et al. [2006].

Theorem 3. An $\mathrm{S}^{3} \mathrm{PMR}$ is live and reversible if no siphon in it can become unmarked Huang et al. [2006].

\section{DEADLOCK PREVENTION POLICY}

Theorem 4. Let $\left(N, M_{0}\right), N=(P, T, F)$, be an $\mathrm{S}^{3} \mathrm{PMR}$ net.(a)As a strongly dependent siphon with $\eta_{S}=\sum_{i=1}^{n} a_{i}$. $\eta_{S_{i}}$, where $S_{1}, S_{2}, \ldots$, and $S_{n}$ are elementary siphons, $S$ is invariant-controlled if (1) $\forall i \in\{1,2, \ldots, n\}, I_{i}$ is a $P$ invariant of $N,\left\|I_{i}\right\|^{+} \subseteq S_{i}$, and $\forall p \in S_{i}, I_{i}(p)=1$; (2) $M_{0}(S)>\sum_{i=1}^{n} \sum_{p \in\left\|I_{i}\right\|^{-}}\left(a_{i} \cdot\left|I_{i}(p)\right| \cdot M_{0}(p)\right)$. (b) $S$ be a weakly dependent siphon with $\eta_{S}=\sum_{i=1}^{n} a_{i} \cdot \eta_{S_{i}}-$ $\sum_{j=n+1}^{n+m} a_{j} \cdot \eta_{S_{j}}$, where $S_{1}, S_{2}, \ldots, S_{n}, S_{n+1}, \ldots$ and $S_{n+m}$ are elementary siphons, $S$ is invariant-controlled if (1) $\forall i \in\{1,2, \ldots, n\}, I_{i}$ is a $P$-invariant of $N,\left\|I_{i}\right\|^{+} \subseteq S_{i}$; (2) $M_{0}(S)>\sum_{i=1}^{n} \sum_{p \in\left\|I_{i}\right\|^{-}}\left(a_{i} \cdot\left|I_{i}(p)\right| \cdot M_{0}(p)\right)$.

Note that the controllability conditions stated in Theorems 4 is sufficient but not necessary. From the basic definition, a siphon $S$ is a potential deadlock if and only if $f(S)=0$, where $f(S)=\min \left\{M(S) \mid M \in R\left(N, M_{0}\right)\right\}$. Therefore, siphon $S$ is said to be controlled if and only if $f(S)>0$. Due to a large number of reachable markings, $f(S)$ is difficult to find. To avoid the difficulty, we consider another function $F(S)$ defined as $F(S)=\min \{M(S)$ $\left.M=M_{0}+[N] \cdot Y, M, Y \geq 0\right\}$, where $M$ and $Y$ are vectors of real numbers. Relation $M=M_{0}+[N] \cdot Y$ is usually called the state equation of $\left(N, M_{0}\right)$. From the basic theory of Petri nets, any reachable marking fulfils the sate equation but the reverse is not true. This implies $F(S)<f(S)$. Hence any siphon with $F(S)>0$ is not a potential deadlock Chu and Xie [1997].

Proposition 1. Let $\left(N, M_{0}\right), N=\bigcirc_{i=1}^{k} N_{i}=\left(P \cup P^{0} \cup\right.$ $\left.P_{R}, T, F\right)$, be a marked S PMR. $\forall S \in \Pi$, add monitor $V_{S}$ and the augmented net is denoted by $\left(N^{\prime}, M_{0}^{\prime}\right)$, where $\forall p \in P \cup P^{0} \cup P_{R}, M_{0}^{\prime}(p)=M_{0}(p), M_{0}^{\prime}\left(V_{S}\right)=M_{0}(S)-\xi_{S}$, $1 \leq \xi_{S}<M_{0}(S)$. $V_{S}$ is added such that $I=p_{x}+\ldots+$ $p_{y}+p_{\alpha}+\ldots+p_{\beta}-V_{S}$ is a $P$-invariant of $N^{\prime}$, where $\left\{p_{x}, \ldots, p_{y}\right\}=S,\left\{p_{\alpha}, \ldots, p_{\beta}\right\}=[\widehat{S}]=\cup_{i=1}^{k}\left\{p \mid p<_{N}\right.$ 
$\left.p^{\prime}, p^{\prime} \in[S] \cap P_{i}, \nexists p^{\prime \prime} \in[S], p^{\prime \prime} \in S P\left(p^{\prime}, p_{i}^{0}\right)\right\}$, and $[\widehat{S}] \cap S=\emptyset$. Then $S$ is invariant-controlled.

Definition 5. Let $\left(N, M_{0}\right)$, be a marked net system and $S$ be a strict minimal siphon of $N, S$ is max-controlled in $N$, iff there exists a P-invariant $I$ such that $\|I\|^{+} \subseteq S,\|I\|^{-} \cap$ $S=\emptyset$ or $\forall p \in\|I\|^{-} \cap S, \max _{p} \bullet=1$, and $I^{T} \cdot M_{0}>$ $\sum_{p \in S}\left[I(p) \cdot\left(\max _{P} \bullet-1\right)\right]$, where $\max _{p} \bullet=\max _{t \in p} \bullet\{W(p, t)\}$.

Definition 6. $\left(N, M_{0}\right)$ is said to be satisfying the maxcontrolled siphon property (max cs-property) iff every strict minimal siphon of $\left(N, M_{0}\right)$ is max-controlled.

Algorithm 1- Deadlock Prevention Policy based on Siphon Control

Let $\left(N, M_{0}\right), N=\bigcirc_{i=1}^{k} N_{i}=\left(P \cup P^{0} \cup P_{R}, T, F\right)$, be a marked $\mathrm{S}^{3} \mathrm{PMR}$.

1) $M_{0}^{\prime}\left(V_{S}\right)=M_{0}(S)-\xi_{S}, \xi_{S}=1$.

2) For any source transitions $t$ of $N$, add an $\operatorname{arc}\left(V_{S}, t\right)$ of weight $W_{p}$, such that $W_{p}>0$, in which $p \in t^{\bullet} \cap P_{i}$.

3) For any transitions $t$ that is not a source transition of $N$, let $p \in \in^{\bullet} t$ and $p^{\prime} \in t^{\bullet}$. Add an $\operatorname{arc}\left(t, V_{S}\right)$ of weight $W_{p}-W_{p^{\prime}}$, if $W_{p}-W_{p^{\prime}}>0$.

Theorem 5. Let $\left(N, M_{0}\right)$ be a marked $S^{3} \mathrm{PMR}$ net and $S$ be a strict minimal siphon of $N, S$ is invariant-controlled after adding monitor $V_{S}$ by Algorithm 1.

Then we develop a method to prevent dependent siphons from being emptied by making its elementary siphons invariant-controlled, which can be achieved by adding monitors to the plant Petri net model.

Algorithm 2 - Deadlock Prevention Policy Based on Elementary Siphons

Let $\left(N, M_{0}\right), N=\bigcirc_{i=1}^{k} N_{i}=\left(P \cup P^{0} \cup P_{R}, T, F\right)$, be a marked $\mathrm{S}^{3} \mathrm{PMR}$.

Step 1) Find the set of elementary siphons $\Pi_{E}$ and the set of dependent siphons $\Pi_{D}$ in $N$. Assume that $\Pi_{E}=\left\{S_{1}, S_{2}, \ldots, S_{m}\right\}$ and $\Pi_{D}=\left\{D S_{1}, D S_{2}, \ldots, D S_{n}\right\}$.

Step 2) By Algorithm 1, add monitors $V_{S_{1}}, V_{S_{2}}, \ldots$, and $V_{S_{m}}$. The extended net system is denoted by $\left(N^{\prime}, M_{0}^{\prime}\right)$, where $\forall i \in$ $\{1,2, \ldots, m\}, M_{0}^{\prime}\left(V_{S_{i}}\right)=M_{0}\left(S_{i}\right)-\xi_{S_{i}}, \xi_{S_{i}}=1$.

Step 3) $\Pi_{D}^{C}:=\emptyset ; \Pi_{D}^{U}:=\emptyset$.

Step 4) $i:=1$.

Step 5) if $i \geq n+1$ then go to Step 6 . $\left\{D S_{i}\right\}$ else if $D S_{i}$ is controlled due to Theorems 4 then $\Pi_{D}^{C}:=\Pi_{D}^{C} \cup$

$$
\begin{aligned}
& \text { else } i:=i+1 \text {; go to Step } 5 \\
& \text { endif } \\
& \text { endif }
\end{aligned}
$$

Step 6) $\Pi_{D}^{U}:=\Pi_{D} \backslash \Pi_{U}^{C}$.

Step 7) Let $\Pi_{D}^{U}=\left\{D S_{1}^{U}, D S_{2}^{U}, \ldots, D S_{k}^{U}\right\}$

Step 8) Let $\Pi_{D}^{U(\alpha)}:=\emptyset$ and $\Pi_{D}^{U(\beta)}:=\emptyset$.

Step 9) $j:=1$.

Step 10) if $j \geq k+1$ then go to Step 11 .

$$
\text { else if } F\left(D S_{j}^{U}\right)>0 \text { then } \Pi_{D}^{U(\alpha)}:=\Pi^{U(\alpha)} \cup\left\{D S_{j}^{U}\right\}
$$

\author{
else $j:=j+1$; go to Step 10 \\ endif \\ endif
}

Step 11) $\Pi_{D}^{U(\beta)}:=\Pi_{D}^{U} \backslash \Pi_{D}^{U(\alpha)}$. Let $\Pi_{D}^{U(\beta)}=\left\{D S_{1}^{U(\beta)}, D S_{2}^{U(\beta)}, \ldots\right.$, $\left.D S_{l}^{U(\beta)}\right\}(l \leq k)$.

Step 12) if $\forall i \in\{1,2, \ldots, m\}, \forall j \in\{1,2, \ldots, l\}, S_{i}$ is an elementary siphon of $D S_{j}^{U(\beta)}$

$$
\begin{aligned}
& \text { then } D S_{j}^{U(\beta)}\left(S_{i}\right)=1 \\
& \text { else } D S_{j}^{U(\beta)}\left(S_{i}\right)=0 \\
& \text { endif }
\end{aligned}
$$

Step 13) Let $\gamma_{i}=\sum_{D S^{U(\beta)} \in \Pi_{D}^{U(\beta)}} D S^{U(\beta)}\left(S_{i}\right), i \in\{1,2, \ldots, m\}$.

Step 14). Let $\gamma_{x}$ be $\max \left\{\gamma_{i} \mid i=1,2, \ldots, m\right\}$, where $x \in$ $\{1,2, \ldots, m\}$.

Step 15) Increase $\xi_{S_{x}}$ until every siphon $S$ in $\left\{D S_{j}^{U(\beta)} \mid D S_{j}^{U(\beta)}\left(S_{x}\right)=\right.$ $1, j \in\{1,2, \ldots, l\}\}$ is either controlled due to Theorems 4 or $F(S)>$ 0.

Step 16) $\Pi_{D}^{U(\beta)}:=\Pi_{D}^{U(\beta)} \backslash\left\{D S_{j}^{U(\beta)} \mid D S_{j}^{U(\beta)}\left(S_{x}\right)=1, j \in\right.$ $\{1,2, \ldots, l\}\}$.

Step 17) if $\Pi_{D}^{U(\beta)}=\emptyset$ then go to Step 19.

Step 18) $\gamma_{x}:=0$; go to Step 14 .

Step 19) Output $\left(N^{\prime}, M_{0}^{\prime}\right)$.

The proposed approach is to add a control place for each strict minimal siphon such that it can never be emptied without generating new strict minimal siphon Ezpeleta et al. [1995]. Note that in Ezpeleta et al. [1995] for every strict minimal siphon $S, \xi_{S}=1$. In this paper, $\xi_{S}$ indicates the least number of tokens that siphon $S$ can hold. Obviously, $\xi_{S}$ is equal to or greater than 1 to achieve a deadlock control purpose. When the above algorithm is applied to an $\mathrm{S}^{3} \mathrm{PMR}$ with $1 \leq \xi_{S}<M_{0}(S)$, all strict minimal siphons in the original net system $\left(N, M_{0}\right)$ are also controlled. Here a controlled strict minimal siphon means that it can never be emptied. On the other hand, since there is no emptiable control-induced minimal siphon in $N^{\prime}$, the siphons of $\left(N^{\prime}, M_{0}^{\prime}\right)$ contain no additional control places, i.e., they are the siphons of the original Petri net model $N$. Therefore, $\max _{p} \bullet=1$ holds. According to the Pinvariant-controlled siphons, $\|I\|^{+} \subseteq S$ and $I^{T} \cdot M_{0}>0$ hold. Hence we have $\sum_{p \in S}\left[I(p) \cdot\left(\max _{P} \bullet-1\right)\right]=0$. We can get immediately that $I^{T} \cdot M_{0}^{\prime}>\sum_{p \in S}\left[I(p) \cdot\left(\max _{P} \bullet-1\right)\right]=0$. Thus $S$ is max-controlled. Based on Algorithm 2, we can verify that a dependent siphon is marked. Therefore, each strict minimal siphon of $\left(N^{\prime}, M_{0}^{\prime}\right)$ is max-controlled and $\left(N^{\prime}, M_{0}^{\prime}\right)$ satisfies the max cs-property.

Theorem 6. The net $\left(N^{\prime}, M_{0}^{\prime}\right)$ is live Algorithm 2.

As mentioned above, a strict minimal siphon $S$ in a marked $\mathrm{S}^{3} \mathrm{PMR}\left(N, M_{0}\right)$ can be invariant-controlled by adding monitor $V_{S}$ such that $S \cup\left\{V_{S}\right\} \cup[\widehat{S}]$ is the support of a P-invariant of the resultant net $\left(N^{\prime}, M_{0}^{\prime}\right)$. The monitor related to $S$ has its output arcs directed to the source transitions of $N$. This is conservative. It can be verified that $\left(N^{\prime}, M_{0}^{\prime}\right)$ could be also live even if we do not make 
all monitors have their output arcs directed to the source transitions of $N$. As shown below, Algorithm 3 can, by re-arranging the output arcs of the control places derived from Algorithm 2, improve the positions of the additional arcs in order that the resultant net remains live and has more permissive behavior than the former. The algorithm is stated as follows.

Algorithm 3 - Optimize the Positions of the Output Arcs

Let $\left(N, M_{0}\right), N=\bigcirc_{i=1}^{k} N_{i}=\left(P \cup P^{0} \cup P_{R}, T, F\right)$, be a marked $\mathrm{S}^{3} \mathrm{PMR}, S$ be a strict minimal siphon of $N$, and $[S]$ be the complementary set of $S$. $\forall i \in\{1,2, \ldots, k\}$, if $[S] \cap P_{i}=\emptyset$, let $B_{S}^{i}=\emptyset$; otherwise, let $B_{S}^{i}=\left\{p \mid \forall S P\left(p_{i}^{0}, p_{\alpha}\right), p \in S P\left(p_{i}^{0}, p_{\alpha}\right) \cap P_{i}, S P\left(p_{i}^{0}, p_{\alpha}\right) \cap\right.$ $\left.[S]=\emptyset, p_{\alpha} \in \bullet p_{\beta}, p_{\beta} \in[S] \cap P_{i}\right\}$. Let $B_{S}=\cup_{i=1}^{k} B_{S}^{i}$. Let $\left\{V_{S_{1}}, V_{S_{2}}, \ldots, V_{S_{m}}\right\}$ be the set of monitors added for the elementary siphons of $N$, and the extended net system is denoted by $\left(N^{\prime}, M_{0}^{\prime}\right)$.

step 1) Derive the set of monitors $\left\{V_{S_{1}}, V_{S_{2}}, \cdots, V_{S_{m}}\right\}$ from Algorithm $2, a:=m$

Step 2) $N_{1}^{\prime}=N^{\prime}, M_{1}^{\prime}=M_{0}^{\prime}$, and $m:=1$

Step 3) if $m \geq a+1$ then $m=m-1$ go to step 11)

else go to step 4)

endif

Step 4) $i:=1$

Step 5) $p:=p_{i}^{0}$

Step 6) $p_{x}:=p$

Step 7) if $i \geq k+1$ then go to Step 10)

else if $B_{S_{m}}^{i}=\emptyset$ then $\mathrm{i}=\mathrm{i}+1$; go to Step 5)

else $B_{S_{m}}^{\triangleleft}:=B_{S_{m}} ; B_{S_{m}}:=B_{S_{m}} \backslash p_{x}^{\bullet \bullet}$

endif

endif

Step 8) Change the output arcs of $V_{S_{m}}$ s.t. $\left[S_{m}\right] \cup B_{S_{m}} \cup V_{S_{m}}$ is the support of a P-invariant, and the resultant net system is supposed to be $\left(N_{m}^{\prime}, M_{m}^{\prime}\right)$.

Step 9) if The resultant net system is live, then change the output $\operatorname{arcs}$ of $V_{S_{m}}$ s.t. $\left[S_{m}\right] \cup B_{S_{m}} \cup V_{S_{m}}$ is the support of a P-invariant of $N_{m}^{\prime}$

$$
\begin{aligned}
& \text { if } \exists p \in B_{S_{m}}, p \in P_{i} \\
& \quad \text { then } \forall p_{y} \in p_{x}^{\bullet \bullet}, p:=p_{y} ; \text { go to Step 6) } \\
& \quad \text { else } i:=i+1 \text {; go to Step 5) } \\
& \quad \text { endif } \\
& \text { else } B_{S_{m}}:=B_{S_{m}}^{\triangle} ; i:=i+1 \text {; go to Step 5) } \\
& \text { endif }
\end{aligned}
$$

Step 10) $m:=m+1$, go to step 3)

Step 11) $N_{1}^{\prime}=N_{m}^{\prime}, M_{1}^{\prime}=M_{m}^{\prime}$

Step 12) Over

Theorem \%. The net $\left(N_{1}^{\prime}, M_{1}^{\prime}\right)$ satisfies the max-controlled siphon property (max cs-property).

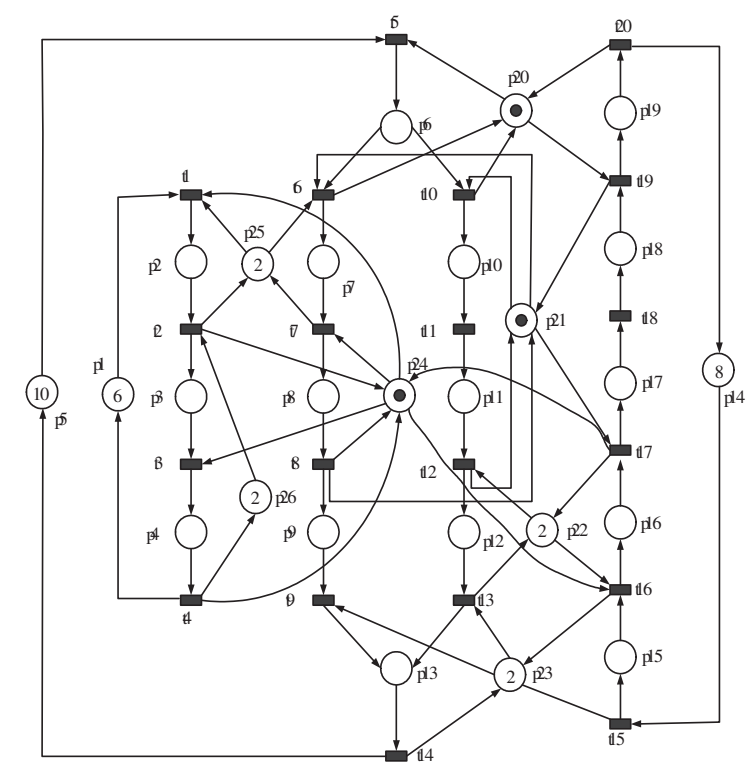

Fig. 1. Petri Net Model for an FMS Cell.

\section{AN FMS EXAMPLE}

Figure 1 shows the Petri net model of an FMS. The net system is an $\mathrm{S}^{3} \mathrm{PMR}$ and contains deadlocks. There are 19 strict minimal siphons and the dependent ones are marked by $*: S_{1}^{*}=\left\{p_{7}, p_{8}, p_{13}, p_{19}, p_{20}, p_{21}, p_{22}, p_{23}\right\}, S_{2}^{*}=\left\{p_{7}, p_{8}, p_{13}\right.$, $\left.p_{17}, p_{18}, p_{21}, p_{22}, p_{23}\right\}, S_{3}=\left\{p_{13}, p_{16}, p_{22}, p_{23}\right\}, S_{4}=\left\{p_{7}, p_{8}\right.$, $\left.p_{10}, p_{11}, p_{19}, p_{20}, p_{21}\right\}, S_{5}^{*}=\left\{p_{7}, p_{8}, p_{12}, p_{19}, p_{20}, p_{21}, p_{22}\right\}$, $S_{6}=\left\{p_{7}, p_{8}, p_{12}, p_{17}, p_{18}, p_{21}, p_{22}\right\}, S_{7}^{*}=\left\{p_{4}, p_{8}, p_{13}, p_{19}\right.$, $\left.p_{20}, p_{21}, p_{22}, p_{23}, p_{24}, p_{26}\right\}, S_{8}^{*}=\left\{p_{4}, p_{8}, p_{12}, p_{19}, p_{20}, p_{21}\right.$, $\left.p_{22}, p_{24}, p_{26}\right\}, S_{9}^{*}=\left\{p_{4}, p_{8}, p_{13}, p_{17}, p_{18}, p_{21}, p_{22}, p_{23}, p_{24}\right.$, $\left.p_{26}\right\}, S_{10}^{*}=\left\{p_{4}, p_{8}, p_{12}, p_{17}, p_{18}, p_{21}, p_{22}, p_{24}, p_{26}\right\}, S_{11}^{*}=\left\{p_{4}\right.$, $\left.p_{8}, p_{11}, p_{19}, p_{20}, p_{21}, p_{24}, p_{26}\right\}, S_{12}^{*}=\left\{p_{4}, p_{8}, p_{11}, p_{17}, p_{18}\right.$, $\left.p_{21}, p_{24}, p_{26}\right\}, S_{13}=\left\{p_{4}, p_{8}, p_{11}, p_{16}, p_{24}, p_{26}\right\}, S_{14}^{*}=\left\{p_{2}, p_{4}\right.$, $\left.p_{8}, p_{13}, p_{19}, p_{20}, p_{21}, p_{22}, p_{23}, p_{24}\right\}, S_{15}^{*}=\left\{p_{2}, p_{4}, p_{8}, p_{12}, p_{19}\right.$, $\left.p_{20}, p_{21}, p_{22}, p_{24}\right\}, S_{16}^{*}=\left\{p_{2}, p_{4}, p_{8}, p_{13}, p_{17}, p_{18}, p_{21}, p_{22}, p_{23}\right.$, $\left.p_{24}\right\}, S_{17}=\left\{p_{2}, p_{4}, p_{8}, p_{12}, p_{17}, p_{18}, p_{21}, p_{22}, p_{24}\right\}, S_{18}^{*}=\left\{p_{2}\right.$, $\left.p_{4}, p_{8}, p_{11}, p_{19}, p_{20}, p_{21}, p_{24}\right\}$, and $S_{19}=\left\{p_{2}, p_{4}, p_{8}, p_{11}\right.$, $\left.p_{17}, p_{18}, p_{21}, p_{24}\right\}$.

The controllability of dependent siphons due to Theorem 4 is shown in Table 1, where DS denotes the dependent siphon. The relationships between the characteristic $T$ vectors of dependent siphons and their elementary siphons are as follows:

$\eta_{1}=\eta_{3}+\eta_{4}+\eta_{6}, \eta_{2}=\eta_{3}+\eta_{6}, \eta_{5}=\eta_{4}+\eta_{6}, \eta_{7}=\eta_{3}+\eta_{4}+\eta_{13}+\eta_{17}$, $\eta_{8}=\eta_{4}+\eta_{13}+\eta_{17}, \eta_{9}=\eta_{3}+\eta_{13}+\eta_{17}, \eta_{10}=\eta_{13}+\eta_{17}, \eta_{11}=$ $\eta_{4}+\eta_{13}+\eta_{19}, \eta_{12}=\eta_{13}+\eta_{19}, \eta_{14}=\eta_{3}+\eta_{4}+\eta_{17}, \eta_{15}=$ $\eta_{4}+\eta_{17}, \eta_{16}=\eta_{3}+\eta_{17}$, and $\eta_{18}=\eta_{4}+\eta_{19}$.

Let us first apply Algorithm 2 to this net system. It is known that there are six elementary siphons. Hence six monitors $V_{S_{3}}, V_{S_{4}}, V_{S_{6}}, V_{S_{13}}, V_{S_{17}}$, and $V_{S_{19}}$ are added to the plant net model. In the plant net, three processes are distinguished with $P_{1}=\left\{p_{2}, p_{3}, p_{4}\right\}, P_{2}=\left\{p_{15}-\right.$ $\left.p_{19}\right\}$, and $P_{3}=\left\{p_{6}-p_{13}\right\}$. Using Algorithm 3, We have $\left[S_{3}\right]=\left\{p_{12}, p_{15}\right\}$. Initially, $B_{S_{3}}=B_{S_{3}}^{1} \cup B_{S_{3}}^{2} \cup B_{S_{3}}^{3}=$ $\left\{p_{6}, p_{10}, p_{11}\right\}$, where $B_{S_{3}}^{1}=B_{S_{3}}^{2}=\emptyset$ and $B_{S_{3}}^{3}=\left\{p_{6}, p_{10}, p_{11}\right\}$. We can begin the algorithm from the output arc $\left(V_{S_{3}}, t_{5}\right)$ with $W\left(V_{S_{3}}, t_{5}\right)=1$. First, let $B_{S_{3}}=\left\{p_{10}, p_{11}\right\}$ and $\left[S_{3}\right] \cup$ $B_{S_{3}} \cup V_{S_{3}}$ be the support of a P-invariant of $N_{1}^{\prime}$. We re- 
Table 1. Marking relationships between dependent and elementary siphons

\begin{tabular}{|c|c|c|}
\hline$D S$ & marking relationships & Ctrl. \\
\hline$S_{1}$ & $M_{0}\left(S_{1}\right)=M_{0}\left(S_{3}\right)+M_{0}\left(S_{4}\right)+M_{0}\left(S_{6}\right)-3$ & $\mathrm{~N}$ \\
\hline$S_{2}$ & $M_{0}\left(S_{2}\right)=M_{0}\left(S_{3}\right)+M_{0}\left(S_{6}\right)-2$ & $\mathrm{~N}$ \\
\hline$S_{5}$ & $M_{0}\left(S_{5}\right)>M_{0}\left(S_{4}\right)+M_{0}\left(S_{6}\right)-2$ & $\mathrm{Y}$ \\
\hline$S_{7}$ & $M_{0}\left(S_{7}\right)=M_{0}\left(S_{3}\right)+M_{0}\left(S_{4}\right)+M_{0}\left(S_{13}\right)$ & $\mathrm{N}$ \\
& $+M_{0}\left(S_{17}\right)-4$ & \\
\hline$S_{8}$ & $M_{0}\left(S_{8}\right)>M_{0}\left(S_{4}\right)+M_{0}\left(S_{13}\right)+M_{0}\left(S_{17}\right)-3$ & $\mathrm{Y}$ \\
\hline$S_{9}$ & $M_{0}\left(S_{9}\right)=M_{0}\left(S_{3}\right)+M_{0}\left(S_{13}\right)+M_{0}\left(S_{17}\right)-3$ & $\mathrm{~N}$ \\
\hline$S_{10}$ & $M_{0}\left(S_{10}\right)>M_{0}\left(S_{13}\right)+M_{0}\left(S_{17}\right)-2$ & $\mathrm{Y}$ \\
\hline$S_{11}$ & $M_{0}\left(S_{11}\right)>M_{0}\left(S_{4}\right)+M_{0}\left(S_{13}\right)+M_{0}\left(S_{19}\right)-3$ & $\mathrm{Y}$ \\
\hline$S_{12}$ & $M_{0}\left(S_{12}\right)>M_{0}\left(S_{13}\right)+M_{0}\left(S_{19}\right)-2$ & $\mathrm{Y}$ \\
\hline$S_{14}$ & $M_{0}\left(S_{14}\right)=M_{0}\left(S_{3}\right)+M_{0}\left(S_{4}\right)+M_{0}\left(S_{17}\right)-3$ & $\mathrm{~N}$ \\
\hline$S_{15}$ & $M_{0}\left(S_{15}\right)>M_{0}\left(S_{4}\right)+M_{0}\left(S_{17}\right)-2$ & $\mathrm{Y}$ \\
\hline$S_{16}$ & $M_{0}\left(S_{16}\right)=M_{0}\left(S_{3}\right)+M_{0}\left(S_{17}\right)-2$ & $\mathrm{~N}$ \\
\hline$S_{18}$ & $M_{0}\left(S_{18}\right)>M_{0}\left(S_{4}\right)+M_{0}\left(S_{19}\right)-2$ & $\mathrm{Y}$ \\
\hline
\end{tabular}

arrange the output arc $\left(V_{S_{3}}, t_{5}\right)$ from $t_{5}$ to $t_{10}$. It can be verified that the addition of $V_{S_{3}}$ by this way produces no emptiable control-induced siphons. Again, let $B_{S_{3}}=\left\{p_{11}\right\}$ and $\left[S_{3}\right] \cup B_{S_{3}} \cup V_{S_{3}}$ be the support of a P-invariant of $N_{1}^{\prime}$. We re-arrange the output arc $\left(V_{S_{3}}, t_{10}\right)$ from $t_{10}$ to $t_{11}$. Similarly, no emptiable control-induced siphon is produced. Now, let $B_{S_{3}}=\emptyset$ and $\left[S_{3}\right] \cup V_{S_{3}}$ be the support of a P-invariant of $N_{1}^{\prime}$. We re-arrange the output arc $\left(V_{S_{3}}, t_{11}\right)$ from $t_{11}$ to $t_{12}$. We can verify that no siphon that can possibly be unmarked is generated due to the re-arrangement. The processing to $V_{S_{3}}$ terminates since in this case, $B_{S_{3}}$ has been empty. Now the incidence relationships between $V_{S_{3}}$ and the transitions can be finalized by the fact that $\left[S_{3}\right] \cup V_{S_{3}}$ is the support of a P-invariant of $N_{1}^{\prime}$.

Table 2. Control performance comparison.

\begin{tabular}{|c|c|c|c|}
\hline control policy & $\begin{array}{c}\text { No. of } \\
\text { monitors } \\
\text { added }\end{array}$ & $\begin{array}{c}\text { No. of } \\
\text { arcs } \\
\text { added }\end{array}$ & $\begin{array}{c}\text { No. of } \\
\text { reachable } \\
\text { states }\end{array}$ \\
\hline Ezpeleta et al. $[1995]$ & 19 & 121 & 2700 \\
\hline Li and Zhou [2004] & 6 & 29 & 2700 \\
\hline The proposed method & 6 & 28 & 3771 \\
\hline
\end{tabular}

Accordingly, by optimizing the positions of the output arcs of monitors $V_{S_{4}}, V_{S_{6}}, V_{S_{13}}, V_{S_{17}}$, and $V_{S_{19}}$, the resultant net, denoted by $\left(N_{1}^{\prime}, M_{1}^{\prime}\right)$ in the case of no confusion, is found as shown in Figure 2, where $M_{1}^{\prime}\left(V_{S_{3}}\right)=3$, $M_{1}^{\prime}\left(V_{S_{4}}\right)=1, M_{1}^{\prime}\left(V_{S_{6}}\right)=2, M_{1}^{\prime}\left(V_{S_{13}}\right)=2, M_{1}^{\prime}\left(V_{S_{17}}\right)=3$, and $M_{1}^{\prime}\left(V_{S_{19}}\right)=1$ by $\xi_{S_{3}}=\xi_{S_{4}}=\xi_{S_{6}}=\xi_{S_{13}}=\xi_{S_{17}}=\xi_{S_{19}}=1$.

Now we check the controllability of dependent siphons. We first deal with $S_{5}$. Note that $\eta_{S_{5}}=\eta_{S_{4}}+\eta_{S_{6}}$. By the way that $V_{S_{4}}$ is added, we can see that $S_{4}$ is controlled by Pinvariant $I_{S_{4}}=p_{7}+p_{8}+p_{10}+p_{11}+p_{19}+p_{20}+p_{21}-V_{S_{4}}$, and $S_{6}$ is controlled by P-invariant $I_{S_{6}}=p_{7}+p_{8}+p_{12}+p_{17}+$ $p_{18}+p_{21}+p_{22}-V_{S_{6}}$. By Theorem 4 , strongly dependent siphon $S_{5}$ is controlled if $M_{1}^{\prime}\left(S_{5}\right)>M_{1}^{\prime}\left(V_{S_{4}}\right)+M_{1}^{\prime}\left(V_{S_{6}}\right)$ holds. Considering $M_{1}^{\prime}\left(S_{5}\right)=M_{0}\left(S_{5}\right)=4, M_{1}^{\prime}\left(V_{S_{4}}\right)=$ $M_{0}\left(S_{4}\right)-\xi_{S_{4}}=2-\xi_{S_{4}}$, and $M_{1}^{\prime}\left(V_{S_{6}}\right)=M_{0}\left(S_{6}\right)-\xi_{S_{6}}=3-$ $\xi_{S_{6}}$, we can say that $S_{5}$ is controlled when $\xi_{S_{4}}=\xi_{S_{6}}=1$. The controllability of $S_{8}, S_{10}, S_{11}, S_{12}, S_{15}$, and $S_{18}$ can be accordingly verified.

There are six equalities about the initial marking relationships in Table 1 . By Theorem 4, we cannot say that $S_{1}, S_{2}$, $S_{7}, S_{9}, S_{14}$, and $S_{16}$ are controlled. If we let $\xi_{S_{3}}=2$, they

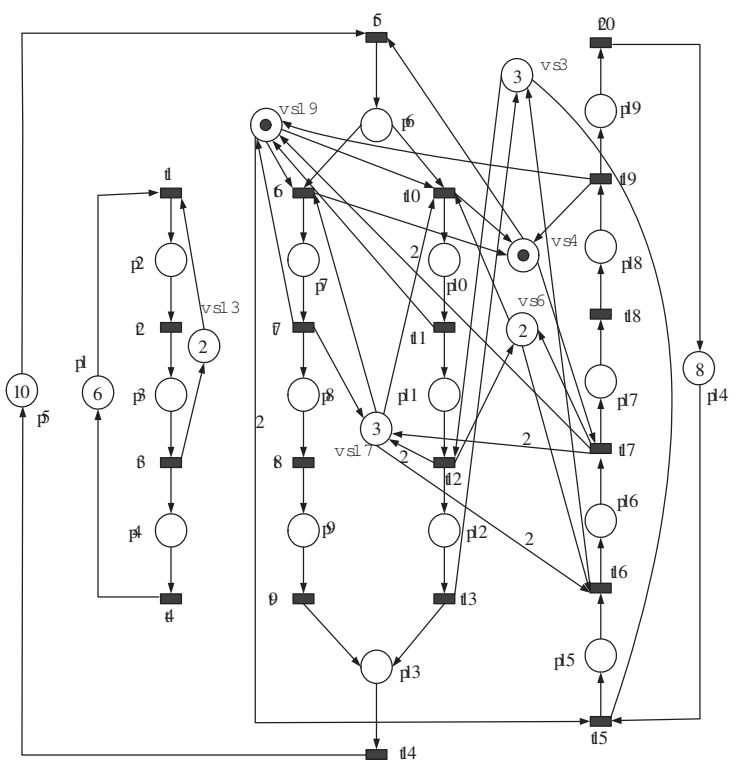

Fig. 2. The supervisor for the FMS without showing the resource places.

become controlled immediately. However, the larger $\xi$, the more behavior is restricted for the controlled system. Note that the six equalities do not necessarily mean that these dependent siphons are uncontrolled since the controllability conditions stated in Theorem 4 are sufficient but not necessary. It is easy to verify that $\forall S \in\left\{S_{1}, S_{2}, S_{7}, S_{9}\right.$, $S_{14}, S_{16}$, we have $F(S)>0$ and that the least number of tokens in them are all two, respectively. Thus, we do not have to increase $\xi_{S_{3}}$ to guarantee the controllability of them. As a result, the net in Figure 2 is live. The number of reachable states, as shown in Table 2, is 3771 . Our deadlock control policy is more permissive than that of Ezpeleta et al. [1995] and Li and Zhou [2004], where, for the same example, both controlled systems have 2700 reachable states, respectively.

\section{CONCLUSION}

This paper presents a deadlock prevention method for a class of FMS, where the deadlocks are caused by the unmarked siphons in their Petri net models. The FMS is modeled using $\mathrm{S}^{3} \mathrm{PMR}$, which is a special class of Petri nets. The major disadvantage of the siphoned-based deadlock prevention methods is that too many monitors have to be added, which leads to a structurally complex liveness enforcing Petri net supervisor, and the behavior of the modelled system seems much restrictive. This paper shows that by adding a small number of monitors to elementary siphons only, all siphons can be prevented from being unmarked. We use a control policy to ensure that by adding a monitor for each elementary siphon, the siphon is successfully controlled and no emptiable control-induced siphons can be produced. In addition, the examples show that our method can achieve much more permissive supervisors than the existing ones Ezpeleta et al. [1995] and Li and Zhou [2004]. 


\section{ACKNOWLEDGMENT}

This work was partially supported by the National Nature Science Foundation of China under Grant No 60474018, the Laboratory Foundation for the Returned Overseas Chinese Scholars, Ministry of Education, PRC, under Grant No 030401, and chang jiang scholars program Ministry of Education, PRC.

\section{REFERENCES}

B. Hruz and M. C. Zhou. Modeling and control of discrete event dynamic systems. Springer, London, UK, 2007.

B. Abdallah and H. A. Elmaraghy. Deadlock prevention and avoidance in FMS: a Petri net based approach. Int J Adv. Manuf. Tech., volume 14, no 10, pages 704-715, 1998.

D. Y. Chao. Computation of elementary siphons in Petri nets for deadlock control. The Computer Journal, volume 49, no 4, pages 470-479, 2006.

F. Chu and X. L. Xie. Deadlock analysis of Petri nets using siphons and mathematical programming. IEEE Trans. Robot. and Automat., volume 13, no 6, pages 793-804, 1997.

F. S. Hsien and S. C. Chang. Dispatching-driven deadlock avoidance controller synthesis for flexible manufacturing systems. IEEE Trans. Robot. and Automat., volume 10, no 2, pages 196-209, 1994.

I. B. Abdallah and H. A. ElMaraghy. Deadlock prevention and avoidance in FMS: a Petri net based approach. International Journal of Advanced Manufacturing Technology, volume 14, no 4, pages 704-715, 1998.

J. Park and S. A. Reveliotis. Deadlock avoidance in sequential resource allocation systems with multiple resource acquisitions and flexible routings. IEEE Trans. Robot. and Automat., volume 46, no 10, pages 1572 1583, 2001.

J. Ezpeleta, J. M. Colom, and J. Martinez. A Petri net based deadlock prevention policy for flexible manufacturing systems. IEEE Trans. Robot. and Automat., volume 11, no 2, pages 173-184, 1995.

K. Barkaoui and I-F. Pradat-Peyre. ON liveness and controlled siphons in Petri nets. Application and Theory of Petri nets., volume 1091, pages 57-72, 1996.

K. Y. Xing, B. S. Hu, and H. X. Chen. Deadlock avoidance policy for Petri-net modelling of flexible manufacturing systems with shared resources. IEEE Trans. Automatic Control., volume 42, no 2, pages 289-295, 1996.

M. P. Fanti and M. C. Zhou. Deadlock control methods in automated manufacturing systems. IEEE Transactions on systems man and cybernetics., Japanese, Part A, volume 34, no 1, pages 5-2, 2004.

M. C. Zhou and M. P. Fanti. Deadlock resolution in computer-integrated systems. Marcel Dekker, January 2005.

M. C. Zhou and V. Kurapati. Modeling, simulation and control of flexible manufacturing fystems: A Petri net approach. World Scientific, Singapore, 1999.

M. C. Zhou and F. DiCesare. Petri net synthesis for discrete event control of manufacturing systems. Kluwer Academic Publishers, Boston, MA, 1993.

M. D. Jeng and X. L. Xie. Deadlock detection and prevention of automated manufacturing systems using Petri nets and siphons. Deadlock Resolution in Computer-
Integrated Systems, M. C. Zhou and M. P. Fanti, New York, Marcel Dekker Co., pages 233-282, 2004.

M. Uzam. An optimal deadlock prevention policy for flexible manufacturing systems using on Petri Net models with resources and the theory of regions. Int. J Adv. Manuf. Syst., volume 19, pages 192-208, 2002.

M. D. Jeng, X. L. Xie, and M. Y. Peng. Process nets with resources for manufacturing modeling and their analysis. IEEE Trans. Robot. and Automat., volume 18, no 6, pages 875-889, 2002.

M. Uzam. An optimal deadlock prevention policy for flexible manufacturing systems using Petri net models with resources and the theory of regions. Int. J. Adv. Manuf. Tech., volume 19, no 3, pages 192-208, 2002.

M. Uzam. The use of Petri net reduction approach for an optimal deadlock prevention policy for flexible manufacturing systems. Int. J. Adv. Manuf. Tech., volume 23, no 3-4, pages 204-219, 2004.

R. A. Wysk, N. S. Yang, and S. Joshi. Detection of deadlocks in flexible manufacturing systems. IEEE Trans. Robot. and Automat., volume 7, no 6, pages 853859, 1991.

T. Murata. Petri nets: properties, analysis, and applications. Proc. IEEE, volume 77, no.4, pages 541-580, 1989.

Y. S. Huang, M. D. Jeng, X. L. Xie, and D. H. Chung. Siphon-based deadlock prevention policy for flexible manufacturing systems. IEEE Trans. Syst., Man, Cybern., volume 36, no 6, pages 2152-2160, 2006.

Y. S. Huang, M. D. Jeng, X. L. Xie, and S. L. Chung. Deadlock prevention policy based on Petri nets and siphons. Int. J. Prod. Res., volume 39, no 3, pages 283305. 2001.

Z. W. Li and M. C. Zhou. Elementary siphons of Petri nets and their application to deadlock prevention in flexible manufacturing systems. IEEE Trans. Syst., Man, Cybern., volume 34, no 1, pages 38-51, 2004.

Z. W. Li and M. C. Zhou. Clarifications on the definitions of elementary siphons of Petri nets. IEEE Trans. Syst. Man, and Cybern, volume 36, no 6, pages 1227-1229, 2006.

Z. W. Li and M. C. Zhou. Two stage methods for synthesizing liveness-enforcing supervisors for flexible manufacturing systems using Petri nets. IEEE Trans. Industrial Informatics, volume 2, no 4, pages 313-325, 2006.

Z. Banaszak and B. H. Krogh. Deadlock avoidance in flexible manufacturing systems with concurrently competing process flows. IEEE Trans. Robot. and Automat, volume 6, no 6, pages 720-734, 1990. 